\title{
Mehr Fortschritt wagen - \\ Der Vertrag der Ampelkoalition zwischen vorsichtiger Erneuerung und rasendem Stillstand
}

\section{FRANZ KNIEPS*}

Franz Knieps ist Vorstand des BKK Dachverbands e.V. und Mitherausgeber der Zeitschrift Gesundheits- und Sozialpolitik

Erstmals in der Geschichte der Bundesrepublik regiert eine Ampelkoalition aus Sozialdemokraten, Bündnisgrünen und Freien Demokraten unter dem neuen Bundeskanzler Olaf Scholz. In disziplinierten Sondierungen und Koalitionsverhandlungen wurde ein 177 Seiten starker Koalitionsvertrag erarbeitet, der die Grundlagen für die politischen Entscheidungen der 20. Legislaturperiode legt. Der Beitrag beschreibt die wesentlichen Inhalte des Kapitels über die Gesundheits- und Pflegepolitik und fragt, was nicht im Vertrag steht. Er sucht nach den strategischen Schwerpunkten und prüft, ob die geplanten Veränderungen geeignet und ausreichend sind, die Herausforderungen an das Gesundheitswesen zu meistern, Schwächen auszumerzen und Potenziale zu erschließen.

\section{Der Machtwechsel und die Folgen für die Gesundheitspolitik}

Eigentlich war der Wahlabend keine echte Überraschung mehr. Nach 16 Jahren Kanzlerschaft von Angela Merkel hatten sich im Laufe des Jahres 2021 Optionen für einen Machtwechsel abgezeichnet, deren Entwicklung hier nicht im Einzelnen nachgezeichnet werden kann (ausführlich Lamby). Auf jeden Fall bestätigte sich wieder eine Erkenntnis von Horst Seehofer, der 1998 nach dem Ende der Regierung Kohl bemerkt hatte: „Mit Gesundheitspolitik kann man keine Wahl gewinnen, aber jede Wahl verlieren.“ Kaum jemand musste diese Erkenntnis schmerzhafter erfahren als der als großer und unerschrockener Reformer gestartete frühere Bundesgesundheits- minister Jens Spahn, den die SARS-Cov 2-Pandemie erst an die Spitze der Beliebtheitsskala der Bundespolitiker führte und der nach etlichen Fehleinschätzungen und operativem Missmanagement einen beispiellosen Absturz erleben musste. Auf der anderen Seite profilierte sich sein Gegenüber Karl Lauterbach als großer Kommunikator zwischen Kassandrarufen und Showeinlagen. (Innerparteiliche) Kritik prallte am Talk Show-Populismus Lauterbachs ebenso ab wie grundsätzliche Zweifel an der Corona-Politik der Großen Koalition (beispielhaft Lütge/ Esfeld; Schularick; Stegemann sowie abwägend die Beiträge in Florack et al.). Hinzu traten Ermüdungserscheinungen

* Der Beitrag gibt ausschließlich die persönliche Auffassung des Autors wieder. 
und interne Zwistigkeiten innerhalb und zwischen den Unionsparteien sowie Zweifel an Auswahlverfahren und Person des Kanzlerkandidaten und eine weitgehende politische Abstinenz der scheidenden Bundeskanzlerin Angela Merkel im Wahlkampf.

Trotz der Pandemie, die in bisher vier Wellen Deutschland, Europa und die Welt überrollt und alle Bereiche von Wirtschaft und Gesellschaft, insbesondere die gesundheitlichen und pflegerischen Einrichtungen, erfasst, hat Gesundheitspolitik außerhalb des Infektionsschutzes im Wahlkampf keine große Rolle gespielt. Zwar haben die Akteure versucht, die Pandemie für ihre ökonomischen und institutionellen Interessen zu nutzen, doch hat sich daraus bisher weder eine konzise Strategie zur Bekämpfung der Pandemie auf der Basis der Erfahrungen von der Spanischen Grippe bis zu HIV-AIDS, SARS, Ebola und Zika (Honigsbaum) noch eine systematische Analyse der Stärken und Schwächen des deutschen Gesundheitswesens und eine grundlegende Debatte um (ordnungspolitische) Alternativen entwickelt (siehe aber die Beiträge bei Hildebrandt/Stuppardt und Klapper/Cichon). Von daher ist es nicht verwunderlich, dass die in einer von 22 thematisch abgegrenzten Arbeitsgruppen erzielten Ergebnisse zu Gesundheit und Pflege kaum öffentlichen Widerhall fanden - mit einer Ausnahme: Die Legalisierung des Verkaufs von Cannabis in speziellen Abgabestellen. Spannend blieb nur die Verteilung und Besetzung der Ressorts. Folglich übersteigen viele Ehrerbietungen an die neue Administration die nüchternen Analysen und Bewertungen der vereinbarten Inhalte.

\section{Was nicht im Koalitionsvertrag steht - Kleinmut statt Strategiewechsel}

Dabei ist es durchaus erstaunlich, was es nicht in den neuen Koalitionsvertrag geschafft hat und was in der Phase zwischen Arbeitsgruppe und finalem Text verändert wurde oder verschwunden ist. Das vielleicht strittigste Thema zwischen SPD und Grünen auf der einen Seite und der FDP auf der anderen Seite wurde schon vor Beginn der Sondierungen von Karl Lauterbach aus dem Weg geräumt.
Das Thema Bürgerversicherung war kein Thema der Gespräche, ohne dass dies auf nennenswerten Widerstand bei deren Protagonistinnen und Protagonisten bei Rot und Grün gestoßen wäre. $\mathrm{Ob}$ damit auch die Erweiterung der Finanzierungsgrundlagen für die Gesetzliche Krankenversicherung - zum Beispiel die Erhöhung von Versicherungspflicht- und Beitragsbemessungsgrenze, die Erfassung weiterer Einkunftsarten oder die

\section{ser weiterer Elakunftsarten oder die}

\section{lest} zahlen müssen, während die Käuferinnen und Käufer von Hunde- und Katzenfutter in den Genuss der Ermäßigung kommen.

Auffällig ist schließlich, dass die Themen Evidenzbasierung und Qualität in dieser Vereinbarung nicht vorkommen. Damit wird eine Chance für eine Schwerpunktsetzung (umfassend Schrappe), aber auch für einzelne Reformteile, wie die die Neuordnung von Krankernhausstrukturen, verspielt. Das ist mehr als ein Symptom dafür, dass die Politik den Diskurs und die Entscheidungsfähigkeit an habilitierte Expertinnen und Experten abgetreten hat und die eminenzblasierte Politikberatung (Hanselle) wieder salonfähig wird. Schließlich hatten Beobachter auch erwartet, dass für Bundesbeamtinnen und -beamte analog dem Hamburger Modell ein Wahlrecht zwischen traditioneller Beihilfe und einem Beitragszuschuss zur sozialen Kranken- und Pflegeversicherung diskutiert würde. Nichts findet sich davon in der endgültigen Vereinbarung.

Das gilt ebenso für zwei weitere grundlegende Punkte, die das Verhältnis von Bund und Ländern in der Planung und Finanzierung von Krankenhäusern sowie die Finanzierung der GKV betreffen. So hatte das Arbeitsgruppenpapier eine Beteiligung des Bundes an der Umgestaltung des stationären Sektors vorgesehen, allerdings unter der Bedingung, dass die Bundesländer bundeseinheitliche Kriterien beachten. Das mochten auch SPD-geführte Bundesländer, speziell die unter Führung ehemaliger Gesundheitsministerinnen stehenden Länder Mecklenburg-Vorpommern und Rheinland-Pfalz, nicht mitmachen. Lieber verzichten sie auf Bundesmittel als endlich der Bevölkerung reinen Wein einzuschenken, dass die erweiterten Möglichkeiten der modernen Medizin unter Fachkräfteund Ressourcenmangel nicht in der heutigen zersplitterten Versorgungslandschaft angeboten werden können. Stattdessen wird die gesamte Problematik in eine „Regierungskommission“ ausgelagert, als

\section{Schwerpunkt Pflege - Ein Sammelsurium von Einzelmaßnahmen statt grundlegender Strukturveränderungen}

Unzweifelhaft lastet der größte Druck von außen und innen auf der Pflege; das gilt sowohl für die Altenpflege als auch für die ambulante und stationäre Krankenpflege. Das gilt für personelle und materielle Ressourcen. Das gilt schließlich für die starke Zersplitterung von medizinischen, pflegerischen und sozialen Leistungen, der unterschiedlichen Leistungsträger und der unzureichenden Finanzierung aus verschiedenen Töpfen. Daher ist es mehr als sinnvoll, dass die Vereinbarungen zu diesem Themenkomplex den Auftakt und den ersten Schwerpunkt des Gesundheitskapitels bilden.

Dabei ist hervorzuheben, dass endlich der größte Pflegedienst der Republik speziell gewürdigt wird. Pflegende Angehörige sollen spürbar entlastet werden. Die Rentenversicherungsbeiträge pflegender Angehörige sollen ebenso wie pandemiebedingte Zusatzausgaben aus Steuermitteln finanziert werden. Leistungen wie Kurzzeit- oder Verhinderungspflege 
sollen in einem sog. Entlastungsbudget zusammengefasst und von bürokratischen Nachweisen entlastet werden. Pflegende Angehörige und Naherstehende sollen mehr Zeitsouveränität enthalten. Im Falle pflegebedingter Auszeiten soll es (erweiterte) Lohnersatzleistungen geben. Speziell für die intensivpflegerische Versorgung soll eine 24stündige Betreuung in der Familie möglich werden.

Zugunsten pflegender Angehöriger dürfte sich die Stärkung kommunaler Mitwirkungs- und Mitbestimmungsmöglichkeiten auswirken. Das gilt vor allem für die Ausweitung quartiernaher Wohnformen und für die Mitgestaltungsmöglichkeiten der Kommunen bei Versorgungsverträgen. Ein Ziel ist der Ausbau der Tages- und Nachtpflege sowie der solitären Kurzzeitpflege. Entlastend für die Familie dürften sich auch die regelhafte Dynamisierung des Pflegegelds und die Begrenzung der Eigenanteile in der stationären Pflege auswirken. Allerdings wird der Koalitionsvertrag wenig konkret, wie dies genau geschehen soll. Es wird nicht ausreichen, die ab 1. Januar 2022 einsetzende prozentuale Bezuschussung zu beobachten und $\mathrm{zu}$ prüfen, wie die Eigenanteile weiter abgesenkt werden können. Das Grunddilemma, dass die Pflegeversicherung nur als Teilkostenversicherung ausgestaltet ist, die ein Abgleiten in die finanzielle Hilfsbedürftigkeit verhindern soll, wird nicht gelöst. Ob die Pflegeversicherung um eine freiwillige und trotzdem paritätisch finanzierte, generationengerechte Vollversicherung ergänzt werden kann, soll eine Expertenkommission klären. Hier scheint die eierlegende Wollmilchsau gesucht zu werden. Nur geringe finanzielle Entlastungen sind von der staatlichen Finanzierung der Rentenversicherungsbeiträge zu erwarten. Stärker ins Gewicht fällt die Übertragung der stationären Behandlungspflege von der Pflegeversicherung auf die Krankenversicherung. Die GKV soll dafür einen pauschalierten Ausgleich erhalten; es bleibt aber unklar, ob das Geld vom Gesundheitsfonds oder von der Pflegeversicherung kommt.

Die Ampelkoalition will die Arbeitsbedingungen der professionell Pflegenden in der Kranken- und Altenpflege schnell und spürbar verbessern. Im Krankenhaus soll die Pflegepersonalregelung 2.0 als Übergangsinstrument einen bedarfsgerechten Qualifikationsmix ermöglichen. Auch in der stationären Langzeitpflege sollen ein neues Personalbemessungsverfahren beschleunigt entwickelt und die Vergütungslücke zur Krankenpflege geschlossen werden. Weitere Maßnahmen sind die Steuerbefreiung von Zuschlägen, die Abschaffung geteilter Dienste, die Einführung trägereigener Springerpools und die Schaffung eines Rechtsanspruchs auf familienfreundliche Arbeitszeiten für Pflegekräfte mit betreuungspflichtigen Kindern. Auf dünnes Eis begibt sich die Ampelkoalition mit der Ankündigung eines erneuten Bonus für Pflegekräfte in besonders belasteten Arbeitssituationen. Hierzu will der Bund die Summe von 1 Mrd. Euro zur Verfügung stellen. Der Bonus soll bis zu einer Höhe von $3000 €$ steuerfrei bleiben. Wie zu erwarten war, entbrannte sofort nach der Ankündigung ein heftiger Streit darüber, wer genau in den Genuss dieser Boni kommen soll und vor allem wer nicht.

Auch die neue Regierung macht es sich zur Aufgabe, das Berufsrechts der Gesundheitsfachberufe zu modernisieren (zu Handlungsfeldern und Möglichkeiten siehe Kühne et al.). Das gilt insbesondere für Pflege- und Hebammenassistenz sowie Rettungssanitäter, aber auch für akademisch ausgebildete Pflegekräfte. Ein neues Berufsbild der „Community Health Nurse“ knüpft an Traditionen der Gemeindeschwester und an positive aktuelle Erfahrungen in Skandinavien oder Kanada an (Inspirierend insbesondere die Beiträge bei Klapper/Cichon). Zudem sollen die Anerkennung ausländischer

\section{Zugunsten pflegender Angehöriger dürfte sich die Stärkung kommunaler Mitwirkungs- und Mitbestimmungsmöglichkeiten auswirken.}

Berufsabschlüsse und die Anwerbung ausländischer Fachkräfte erleichtert und beschleunigt werden. Gespannt sein darf man auf ein allgemeines Heilberufegesetz, nicht nur wegen bei der Frage, wie es um die Zukunft der Heilpraktiker bestellt ist. Das Thema Direktzugang zu therapeutischen Berufen soll erst einmal auf ein Modellprojekt begrenzt werden. Erneut also ein Spiel auf Zeit statt mutiger Schritte nach vorn.

\section{Kleine Reformschritte statt Paradigmenwechsel - Die Prolongierung des versäulten Versorgungssystems}

Wer eine grundlegende Entscheidung für eine integrierte, patientenzentrierte Versorgung erwartet hatte, der wurde vom Koalitionsvertrag nur unzureichend bedient. Auch wenn dieser viele kleine Schritte zu einer besseren Verzahnung enthält, der von Wissenschaft und Praxis angemahnte Paradigmenwechsel zur Überwindung der Sektorengrenzen (Exemplarisch Piwernetz/Neugebauer; Hermann et al.) ist erneut ausgeblieben, selbst wenn der 2. Satz im Gesundheitskapitel des Vertrags genau dieses Ziel formuliert. Die Instrumente - wie eine sektorenübergreifende Versorgungsplanung und eine sektorengleiche Vergütung für ambulant-sensitive Leistungen - sind sicher ebenso positiv $\mathrm{zu}$ bewerten wie der Ausbau multiprofessioneller, integrierter Gesundheits- und Notfallzentren zur flächendeckenden, wohnortnahen Versorgung mit bedarfsgerechten ambulanten und kurzstationären Angeboten. Ob die Notfallversorgung durch die Kassenärztlichen Vereinigungen oder durch die Betreiber der Zentren sichergestellt wird, kann in Abstimmung mit dem Land vor Ort entschieden werden. Ob sich die Absicht, die KV-Notdienste mit den Rettungsleitstellen zu verschränken, realisieren lässt, dürfte wohl von dem Verhalten der Landesinnenminister abhängen. Sie werden sich auch die geplante Eingliederung des Rettungswesens als integrierter Leistungsbereich in das SGB V wohl einiges kosten lassen. Inwieweit die Erweiterung des Spielraums für innovative Versorgungsformen und speziell für regionale bevölkerungsbezogene Verträge wirklich zur Integration beitragen kann, hängt von der Ausgestaltung der ökonomischen Anreize und den Freiheitsgraden zur Abbildung regionaler Besonderheiten ab. Vor allem dürfen die Lordsiegelbewahrer des Kollektivvertrags nicht länger zum Bremser innovativer Versorgungsformen werden. Das ist auch bei der geplanten Verstetigung des Innovationsfonds zu beachten. Hier haben die Koalitionäre zutreffend erkannt, dass die Regelungen 
zur Überführung erfolgreicher Projekte in die Regelversorgung nachjustiert werden müssen.

Noch größer sind die Zweifel vieler Betrachter, ob bei Krankenhausplanung und -finanzierung wirkliche Veränderungen gewollt sind. Allzu vage sind die Vorstellungen für einen Bund-Länder-Pakt über eine moderne und bedarfsgerechte Krankenhausversorgung formuliert. Gegen Leitplanken für eine auf Leistungsgruppen und Versorgungsstufen basierende und sich an Kriterien wie Erreichbarkeit und demographischer Entwicklung orientierter Kapazitätsplanung ist wenig einzuwenden. Das steht schon explizit in den meisten Landeskrankenhausgesetzen, doch die Realität sieht anders aus. Folglich wundert es nicht, dass dem Bund Einflussnahmen durch Anreizsysteme wie einem Restrukturierungstopf verwehrt bleiben. Und die Formulierung zur Weiterentwicklung der Krankenhausfinanzierung durch die Ergänzung um ein nach Versorgungsstufen differenziertes System erlösunabhängiger Vorsorgepauschalen weckt bei den Krankenkassen die Befürchtung, dass hier erneut Kosten von den zuständigen Bundesländern auf die Kostenträger verschoben werden sollen. Es bedarf keiner großen Phantasie, was hier die kurzfristig einzusetzende „Regierungskommission“

\section{Die Zweifel vieler Betrachter sind groß, ob bei Krankenhausplanung und -finanzierung wirkliche Veränderungen gewollt sind.}

ärztlichen Vereinigungen festhalten, aber Kommunen und Ländern neue Mittel gegen Unterversorgung an die Hand geben. So soll die Gründung kommunaler Medizinischer Versorgungszentren erleichtert werden. Die Länder sollen künftig bei Entscheidungen der Zulassungsausschüsse mitwirken können. Eher symbolische Wirkung dürfte die Aufhebung der Budgetierung für hausärztliche Honorare in unterversorgten Regionen, haben, denn der überwiegende Teil dieser Honorare ist heute schon pauschaliert oder entdeckelt.

Einen großen Erfolg können Psychotherapeuten und Psychiater verbuchen. Der Koalitionsvertrag enthält ein deutliches Bekenntnis zur Entstigmatisierung psychischer Erkrankungen und zum Ausbau einer bedarfsgerechten psychotherapeutischen Versorgung. Dazu soll die Bedarfsplanung für Psychotherapeuten mit dem Ziel reformiert werden, die Wartezeiten vor einer Behandlung speziell für Kinder und Jugendliche sowie in ländlichen und strukturschwachen Regionen zu verkürzen. Die ambulante Versorgung von Patienten mit schweren und komplexen Erkrankungen sollen ebenso wie die psychiatrische Notfall- und Krisenversorgung verbessert werden. Der Zugang zu ambulanten Komplexleistungen soll gewährleistet werden. Im stationären Sektor stehen die Orientierung an Leitlinien und die Gewährleistung einer bedarfsgerechten Personalausstattung im Vordergrund.

Die Ampelkoalition will das Präventionsgesetz weiterentwickeln und die Primär- und Sekundärprävention stärken. Erfreulich ist, dass sie Präventi-

vorschlagen wird. Ähnlich dürfte auch das Versprechen zu verstehen sein, kurzfristig für eine bedarfsgerechte auskömmliche Finanzierung von Geburtshilfe, Pädiatrie und Notfallversorgung zu sorgen. Das riecht nach Selbstkostendeckung. So wird das DRG-System, dass keinesfalls frei von Mängeln und Fehlanreizen ist, sturmreif geschossen. Und von Qualität, dem Metathema der beiden letzten Koalitionsverträge, ist an keiner Stelle des Vertrags die Rede. Längst überfällig ist der Ausschluss der Zahlung von Weiterbildungsanteilen im DRG-System an Kliniken, die nicht ausbilden.

In der ambulanten ärztlichen Versorgung will die Koalition zwar grundsätzlich am Sicherstellungsauftrag für die Kassen- on als umfassende gesamtgesellschaftliche Aufgabe sieht. Ob es dazu eines eigenen Nationalen Präventionsplans bedarf, kann dahin gestellt bleiben. Es bist auf jeden Fall sinnvoll, sich näher mit den beispielhaft angeführten Themen wie Diabetes, Alterszahngesundheit, klima- und umweltbedingte Erkrankungen, Einsamkeit oder Suizid zu befassen. Nicht jedes ständigkeitsbereich der Krankenkassen. Als puren Populismus ist die Ankündigung zu bewerten, die Möglichkeiten für Werbemaßnahmen und Werbegeschenke durch Krankenkassen zu beschränken. Damit kein Missverständnis entsteht: Es ist nicht Aufgabe einer sozialen Krankenversicherung, Profisport zu unterstützen. dieser Themen fällt allerdings in den $\mathrm{Zu}$ -
Wer aber schon beim ersten Shitstorm vor dem organisierten Sport einknickt, sollte endlich aufhören, Detailregelungen für Art und Umfang von Werbemaßnahmen in einer wettbewerblichen Krankenversicherung zu treffen. Auch in der Ampelkoalition scheint unklar, welche Rolle Wahlfreiheit und Wettbewerb im Gesundheitswesen spielen sollen und welchen Grad an Differenzierung die Politiker ertragen will. In Sachen Ordnungspolitik muss man leider wieder einmal Fehlanzeige diagnostizieren.

\section{Public Health vor einer Renaissance? - Lehren aus der Pandemie}

Die Pandemie hat Stärken und Schwächen des deutschen Gesundheitswesens wie unter einem Brennglas sichtbar gemacht (Klemm/Knieps). Das gilt in besonderem Maße für die Ausblutung des Öffentlichen Gesundheitsdienstes (ÖGD), wofür vor allem die Länder die Verantwortung tragen. Aber auch das Zusammenspiel von Bund, Ländern und Kommunen hat den Stresstest der Pandemie nicht bestanden. Folglich ist es hilfreich, dass der Bund sein institutionelles und finanzielles Engagement ausbaut, auch wenn es Zweifel gibt, ob er hierfür überhaupt zuständig ist. Augenfällig wurde zudem, dass Pandemiepläne zwar in der Schublade schlummerten, aber deren Umsetzung unzureichend geprobt wurde und im Ernstfall nicht erfolgte. Schließlich fehlte es an der Bevorratung von Arzneimitteln und Medizinprodukten. All dem will die neue Koalition mit einem Ebenen übergreifenden Pakt für den ÖGD und einem Gesundheitssicherstellungsgesetz entgegenwirken.

Die Bundeszentrale für gesundheitliche Aufklärung (BZgA), die sich große Verdienste bei der Bekämpfung von HIVAids erworben hatte, glänzt in der SARSCOV 2-Pandemie durch Funkstille. Auch die Krisenkommunikation des Robert Koch Instituts (RKI) lässt zu wünschen übrig. Außerdem werden Zweifel an der wissenschaftlichen Unabhängigkeit des RKI und der Integration multidisziplinärer Public Health-Ansätze geäußert. Die Ampelkoalition will deshalb ein Institut für öffentliche Gesundheit schaffen, das alle Aktivitäten im Public Health-Bereich, die Vernetzung des ÖGD und die Gesundheitskommunikation des Bundes bündeln soll. 
Da Organisationsveränderungen in der Regel maximalen Widerstand der Betroffenen auslösen, geht die Politik solche Veränderungen eher vorsichtig an. So sollen Selbstverwaltungsstrukturen der Pflege nach Vorstellungen der Pflegekräfte aufgebaut und deren adäquate Mitwirkung an Entscheidungen des Gemeinsamen Bundesausschusses (G-BA) gewährleistet werden. Die Unabhängige Patientenberatung (UPD) soll in eine dauerhafte, staatsferne und unabhängige Struktur unter Beteiligung der maßgeblichen Patientenorganisationen überführt werden. Eine Stiftungslösung ist greifbar nahe.

\section{Die digitale Transformation im Gesundheitswesen - Ein unterschätzter Treiber von Veränderungen}

Die Ampelkoalition kündigt eine regelmäßig fortzuschreibende Digitalisierungsstrategie im Gesundheitswesen an. Diese soll der Lösung von Versorgungsproblemen dienen und nutzerorientiert sein, Die digitale Transformation soll nicht nur in der Pflege der Begrenzung des Dokumentationsaufwands dienen sowie soziale Teilhabe und vielfältige therapeutische Anwendungen fördern. Der Koalitionsvertrag bezieht sich dabei auf die die bekannten Felder telemedizinischer Anwendungen, stößt aber keine neuen Türen auf, hinter denen - wie bei der Entwicklung der personalisierten Medizin auf der Basis von Genomsequenzierung - komplexe ethische, rechtliche und ökonomische Fragen lauern. Deutlich ambitionierter ist der allgemeine Teil des Koalitionsvertrags zu digitalen Innovationen und digitaler Infrastruktur. Aber dort sind Abstimmungsprobleme zwischen den Parteien und den Ressorts zu erwarten. Gleiches gilt im Übrigen beim Thema Förderung der Gesundheitswirtschaft im Kapiteln Wirtschaft.

Löblich ist die Absicht, die Einführung der elektronischen Patientenakte (ePA) und des elektronischen Rezepts $\mathrm{zu}$ beschleunigen. Dazu ist es aber erforderlich, die Aufgaben und Rollen der gematik, die die neue Regierung in eine digitale Gesundheitsagentur ausbauen will, genauer zu beschreiben und mehr Sachverstand aus der Praxis einzubeziehen. Die Politik folgt der Empfehlung des Sachverständigenrats zur Begutachtung der Entwicklung im Gesundheitswesen (SVR), allen Versicherten eine ePA zur Verfügung zu stellen; deren Nutzung bleibt allerdings freiwillig. Datenschutzkonform soll die bisherige Opt-in-Lösung durch ein Opt-out ersetzt werden. Außerdem sollen sämtliche Akteure an die Telematikinfrastruktur angeschlossen werden.

Die Ampelkoalitionäre kündigen ein Registergesetz und ein Gesundheitsdatennutzungsgesetz zur besseren wissenschaftlichen Nutzung der Gesundheitsdaten an. Dass diese Vorhaben mit der Europäischen Datenschutzgrundverordnung (DGSVO) in Einklang stehen müssen, ist selbstverständlich. Man wird jedoch die Umsetzung kritisch daraufhin zu überprüfen haben, inwieweit auch private Dritte Zugriff auf diese Daten erhalten sollen. Es kann nicht angehen, dass neue Geschäftsmodelle der Industrie auf der kostenlosen Nutzung von öffentlichen Daten aufbauen, die Daten gegebenenfalls den europäischen Rechtsraum zur Verarbeitung verlassen und dann zur Schaffung erheblicher Gewinnoptionen teuer an die öffentlichen Gesundheitssysteme in Europa zurückverkauft werden. Hier müssen die öffentlichen Akteure aber auch die Datenschutzbeauftragten des Bundes und der Länder aufmerksam sein und gegebenenfalls entschlossen Widerstand leisten. Sie müssen aber auch ihre digitale Lethargie aufgeben und entschlossen eigene Aktivitäten zur Erschließung, Analyse und Nutzung ihrer Daten auf öffentlichrechtlichen Plattformen starten.

Leider beschränkt sich die angekündigte Durchforstung des SGB V und anderer Normen auf die Überprüfung von durch den technischen Fortschritt überholten Dokumentationspflichten. Fast jede Regierung hat ein Paket zum Abbau von Bürokratie im Gesundheitswesen angekündigt, im Laufe der Legislatur dann aber unzählige neue Vorschriften erlassen. Diese waren nicht selten im Eilverfahren durchgepeitschte Regulierungen, oft in sich widersprüchlich und unzureichend aufeinander abgestimmt (Knieps). Daher wäre es sinnvoll, das gesamte Gesundheitsrecht neu zu kodifizieren, das Leitbild des SGB V zu modernisieren sowie Regulierungsbreite und Regulierungsdichte zu reduzieren (Robert Bosch Stiftung).

\section{Wer soll das bezahlen? - Die Leerstellen bei der Finanzierung}

Sehr schmallippig wird die neue Koalition, wenn es um die Frage geht, wie die expandierenden Gesundheitsausgaben denn finanziert werden sollen. Diese sind übrigens weniger der Pandemie, sondern mehr dem Gebaren der früheren Minister Gröhe und Spahn geschuldet. Die beiden Unionsminister hatten jeden Konflikt mit Geld zugeschüttet und Kostendämpfung aus dem Arsenal der Gesundheitspolitik verbannt. Da hilft es wenig, dass der Koalitionsvertrag ein wuchtiges Bekenntnis zur stabilen und verlässlichen Finanzierung der GKV abgibt. Nur für das Jahr 2022 ist diese Stabilität - dank erhöhter Bundeszuschüsse - weitgehend gesichert. Ab 2023 drohen dann beträchtliche Erhöhungen der Zusatzbeitragssätze. Das noch von der alten Regierung abgegebene Versprechen zur Einhaltung der imaginä-

Bei der Lektüre des Kapitels Gesundheit und Pflege bleibt der unschöne Gesamteindruck, dass dort das Motto „Mehr Fortschritt wagen" nur unzureichend umgesetzt wird.

ren $40 \%$-Grenze für die Sozialabgaben scheint obsolet. Eine moderate Erhöhung der Beitragssätze zur Pflegeversicherung kündigt der Vertrag bereits an. Ob das spontan gegebene Versprechen des neuen Ministers am Tage seiner Ernennung, man werde keine Leistungskürzungen vornehmen, vier Jahre hält, werden schon die Entscheidungen zur Sicherung der Finanzierung ab dem Jahr 2023 zeigen.

Zwar soll der Steuerzuschuss des Bundes verstetigt werden, aber es ist unklar, in welcher Höhe. Auch die Formulierung zur Finanzierung von Beiträgen für Bezieherinnen und Bezieher von Arbeitslosengeld II, das ja durch ein Bürgergeld ersetzt werden soll, wirft Fragen auf. Schon die frühere Große Koalition hatte eine ähnliche Formulierung in ihrem Koalitionsvertrag. Gescheitert ist dessen Umsetzung am Widerstand der SPD-geführten Ressorts für Finanzen sowie für Arbeit und Soziales. Misstrauen erweckt auch der Bezug auf „höhere“ Beiträge. Das weckt Befürchtungen, dass nur Beitragssatzerhöhungen für diese Personengruppe steuerfinanziert 
werden sollen, nicht aber die schon heute unzulängliche Grundfinanzierung. Einen Tropfen auf den heißen Stein bildet das Anschärfen der Regelung zur Preisbildung von Arzneimitteln. Der Herstellerrabatt bleibt, und die Zeit für eine Markteinführung ohne Preisregulierung wird von 12 auf 6 Monate verkürzt. Was sich noch hinter der „Stärkung der Möglichkeiten für Krankenkassen zur Begrenzung der Arzneimittelpreise“ verbirgt, bleib abzuwarten. Und zur Halbierung des Mehrwertsteuersatzes auf Arzneimittel konnte sich die Koalition nicht durchringen. Das wird die Hersteller freuen, denn sie gründen ihre Geschäftsmodelle teilweise auf die Erstattung der Umsatzteuer in der Lieferkette.

Auch wenn dem Koalitionsvertrag kein aussagefähiges Finanztableau beigefügt ist, kann man unschwer erkennen, dass die dürftigen Regelungen zur Finanzierung keineswegs ausreichen, um die Dynamik des Ausgabenanstiegs in der GKV aufzufangen. Im Gegenteil:
Die anvisierten Leistungsverbesserungen und (notwendigen) Investitionen in den Umbau des Gesundheitswesens - auch zur Vorsorge vor künftigen besonderen Ereignissen - werden mehr Geld kosten. Dabei ist zudem zu beachten, was sich aus den Veränderungen im Kapitel zu Arbeit und Sozialstaat ergibt. Die dort anvisierten Änderungen zur Entlastung von Selbständigen werden zu Mindereinnahmen in den Kassen der Sozialversicherungsträger führen.

\section{Ein zwiespältiger \\ Gesamteindruck - Ist das Glas halbvoll oder halbleer?}

Nicht zuletzt deshalb bleibt bei der Lektüre des Kapitels Gesundheit und Pflege der unschöne Gesamteindruck, dass dort das Motto „Mehr Fortschritt wagen“ nur unzureichend umgesetzt wird. Manche der wesentlichen Strukturprobleme werden erst gar nicht angegangen (Zusammenfassend Hermann et al.) Bei anderen will viel man zwar einige Trippelschritte gehen, vermeidet aber mutige Veränderungen aus Furcht vor Zumutungen und Belastungen. Die wurden wegverhandelt, in Kommissionen verbannt oder auf unverbindliche Prüfaufträge reduziert. Auch bleiben viele Maßnahmen, die zweifellos Potenzial für Versorgungsverbesserungen enthalten, ohne realistische Finanzierungsperspektive. Selbst wenn ein Umbau von Strukturen oder eine Effizienzsteigerung in Prozessen rentabel erscheint, muss erst einmal investiert werden. Noch immer werden gleichzeitig Sonderwünsche und Forderungen einzelner Akteure im Gesundheitswesen erfüllt. Ein Wille zur Kostendämpfung ist (noch) nicht vorhanden. Ob damit in der Gesamtbewertung das Glas halbvoll oder halbleer ist, liegt im Auge des Betrachters. Für einen optimistischen Rheinländer ist die Frage leicht zu beantworten. Ob diese Antwort nach vier Jahren einem Faktencheck standhalten wird, weiß heute niemand.

\section{Literatur}

Robert Bosch Stiftung (Hrsg.), Neustart für das Gesundheitsrecht - Ein Handlungskatalog für Politik und Selbstverwaltung, Eigenverlag, Stuttgart 2021

\section{Martin Florack/Karl-Rudolf Korte/} Julia Schwanholz (Hrsg.), Coronakratie Demokratisches Regieren in Ausnahmezeiten, Campus Verlag, Frankfurt/Main 2021

Ralf Hanselle, Postpolitisches Pandemiepalaver, Cicero Online vom 15.12.2021

Christopher Hermann/Franz Knieps/ Hartmut Reiners, Schwerpunkte einer Gesundheitspolitik für die neue Wahlperiode, GuS 4-5/2021, 6-11

Helmut Hildebrandt/Rolf Stuppardt (Hrsg.)

Zukunft Gesundheit - regional, vernetzt, patientenorientiert, medhochzwei Verlag, Heidelberg 2021

Mark Honigsbaum, Das Jahrhundert der Pandemien, Piper Verlag, München 2021
Bernadette Klapper/Irina Cichon (Hrsg.), Neustart! - Für die Zukunft unseres Gesundheitswesens, Medizinisch Wissenschaftliche Verlagsgesellschaft, Berlin 2021

Anne Klemm/Franz Knieps, Unter dem Corona-Brennglas: Erste Lehren aus der Pandemie, GuS 4-5/2020, 67-73

Franz Knieps, Brauchen wir eine Generalüberholung des SGB V? - Perspektiven für eine Neukodifizierung, GuS 1/2020, 75-82

Roy Kühne/Jürgen Graalmann/Franz Knieps (Hrsg.), Die Zukunft der Gesundheits(fach)berufe, Medizinisch Wissenschaftliche Verlagsgesellschaft, Berlin 2021

Stephan Lamby, Entscheidungstage - Hinter den Kulissen des Machtwechsels, C.H.Beck Verlag, München 2021

Christoph Lütge/Michael Esfeld, Und die Freiheit? - Wie die Corona-Politik und der Missbrauch der Wissenschaft unsere offene
Gesellschaft bedrohen, riva Verlag, München 2021

Klaus Piwernetz/Edmund Neugebauer, Strategiewechsel jetzt! - Corona-Pandemie als Chance für die Neuausrichtung unseres Gesundheitswesens, Walter de Gruyter Verlag, Berlin 2021

Sachverständigenrat zur Begutachtung der Entwicklung im Gesundheitswesen, Digitalisierung für Gesundheit - Ziele und Rahmenbedingungen eines dynamisch lernenden Gesundheitssystems, Eigenverlag, Berlin/Bonn 2021

Matthias Schrappe, Qualität 2030 - Die umfassende Strategie für das Gesundheitswesen, Medizinisch Wissenschaftliche Verlagsgesellschaft, Berlin 2015

Moritz Schularick, Der entzauberte Staat Was Deutschland aus der Pandemie lernen muss, C.H.Beck Verlag, München 2021

Bernd Stegemann, Die Öffentlichkeit und ihre Feinde, Klett-Cotta Verlag, Stuttgart 2021 